\title{
Estado nutricional e consumo de energia e nutrientes de pré- escolares que frequentam creches no município de Manaus, Amazonas: existem diferenças entre creches públicas e privadas?
}

\author{
Nutritional status and energy and nutrients intake of children attending day-care centers in the city of \\ Manaus, Amazonas, Brazil: are there differences between public and private day-care centers?
}

Bruno Mendes Tavares' ${ }^{1}$, Gloria Valeria da Veiga ${ }^{2}$, Lucia Kiyoko O. Yuyama ${ }^{3}$, Milena Baptista Bueno $^{4}$, Regina Mara Fisberg ${ }^{5}$, Mauro Fisberg ${ }^{6}$

\section{RESUMO}

Objetivo: Verificar o estado nutricional e o consumo alimentar de crianças assistidas em creches públicas e privadas no município de Manaus, Amazonas.

Métodos: Foram avaliadas crianças entre 24 e 72 meses de duas creches públicas $(n=217)$ e duas creches privadas $(n=91)$ de Manaus. O estado nutricional foi classificado pelos índices peso para idade, peso para estatura, estatura para idade e índice de massa corporal (IMC) para idade, em valores de escores Z. O consumo alimentar na creche foi avaliado pelo método da pesagem direta individual dos alimentos e, no domicílio, pelo registro alimentar de um dia aplicado aos responsáveis. Estimou-se a frequência de crianças com ingestão de nutrientes acima ou abaixo dos pontos de corte de Estimated Average Intake (EAR) ou Adequate Intake (AI).

Resultados: Verificou-se maior frequência de crianças com excesso de peso nas creches privadas segundo os índices peso para estatura e IMC para idade. As crianças das instituições públicas, quando comparadas àquelas das privadas, consumiram mais gorduras poli-insaturadas, trans, ácido graxo ômega-6, vitamina $\mathrm{C}$ e sódio, e menos zinco. Em ambos os tipos de creches observaram-se consumo elevado de energia e proporção elevada de crianças com consumo de vitaminas A e C, zinco e sódio acima do limite superior tolerável de ingestão. A proporção de crianças com inadequação de consumo de cálcio foi maior nas creches públicas do que nas privadas $(27,6$ versus $7,9 \% ; p<0,001)$.

Conclusões: $\mathrm{O}$ consumo de energia e de sódio foi elevado em ambos os tipos de creche e o consumo de cálcio, insuficiente, principalmente nas creches públicas. Recomenda-se melhor monitoramento da dieta infantil para prevenção de doenças futuras.

Palavras-chave: pré-escolar; estado nutricional; consumo de alimentos; creches.

\section{ABSTRACT}

Objectives: To assess the nutritional status and dietary intakes of children attending public and private day care centers in Manaus, Brazil.

Methods: The study assessed children aged 24 to 72 months, enrolled at two public $(n=217)$ and two private

Endereço para correspondência

Gloria Valeria da Veiga

Centro de Ciências da Saúde, Bloco J - $2^{\circ}$ andar

Av. Carlos Chagas Filho 373 - Ilha do Fundão

CEP 21941-590 - Rio de Janeiro/RJ

E-mail: gvveiga@globo.com

Fonte financiadora: O projeto recebeu apoio parcial da Danone Research para financiamento de passagens para o treinamento das equipes do estudo multicêntrico Nutri Brasil Infância e compra de materiais

Conflito de interesse: Mauro Fisberg presta, eventualmente, assessoria nutricional, desenvolve conferências ou realiza projetos de pesquisa para Abbott, Danone, CDN, Sanofi, Wyeth, Nestlé, Kraft, sem vínculos com quaisquer das empresas. A empresa Danone financiou parcialmente a pesquisa e em nenhum momento interferiu no desenho, realização ou analise do estudo

Recebido em: 7/2/2011

Aprovado em: 1/7/2011 
$(\mathrm{n}=91)$ day care centers in Manaus. Nutritional status was classified according to $Z$ scores for weight-for-age, weightfor-height, height-for-age and BMI-for-age. Dietary intakes were measured using the direct food-weighing method for 1 day. A 1-day dietary recall was administered to parents to assess dietary intakes outside the day care centers. The frequencies of children with nutrient intakes above and below the Estimated Average Requirements (EAR) or Adequate Intake (AI) cutoffs were calculated.

Results: There proportion of overweight children was higher at the private day care centers, according to both weight-for-height and BMI-for-age indexes. Children from the public day care centers had higher intakes of polyunsaturated fat, trans fat, omega- 6 , vitamin $\mathrm{C}$ and sodium, and lower zinc intake when compared to those at private centers. Elevated energy intake and a high proportion of children with vitamin $A$, vitamin $C$, zinc and sodium intakes over the Tolerable Upper Intake Levels were observed at both types of day care center. There was a higher proportion of children with inadequate calcium intake at the public centers (27.6 vs. $7.9 \%, p<0.001)$.

Conclusion: Energy and sodium intakes were above recommended levels at both types of day care center. Calcium intakes were below recommended, especially at the public day care centers. Children's diets should be monitored more closely to prevent diseases later in life.

Key-words: child, preschool; nutritional status; food consumption; child day care centers.

\section{Introdução}

O consumo adequado de vitaminas e minerais na infância é fundamental para evitar carências nutricionais que prejudicarão o crescimento. A desnutrição energéticoproteica, a anemia ferropriva e a deficiência de vitamina A representam os principais problemas para a saúde infantil $^{(1)}$. No Brasil, a desnutrição energético-proteica avaliada com base no índice estatura-para-idade, embora em declínio, ainda acomete $6,0 \%$ das crianças menores de cinco anos, chegando a atingir $8,5 \%$ na Região Norte ${ }^{(2)}$. Por outro lado, o excesso de peso foi observado em aproximadamente um quarto das crianças menores de seis anos assistidas em instituições públicas e privadas de nove estados brasileiros ${ }^{(3)}$.

Segundo dados da Pesquisa Nacional de Demografia e Saúde realizada em 2006, a prevalência de anemia é de
20,9\%, enquanto a hipovitaminose A está presente em 7,4\% das crianças menores de cinco anos ${ }^{(4)}$. Na Região Amazônica, a anemia ferropriva foi observada em até $30,6 \%$ de préescolares $^{(5)}$, assim como já foram descritas nesta faixa etária desnutrição crônica, consumo insuficiente de niacina, ferro e vitamina $A^{(6)}$.

A maioria dos estudos sobre tais carências foi desenvolvida em crianças assistidas em creches, as quais podem ter um impacto positivo sobre o seu estado nutricional, principalmente para aquelas pertencentes a estratos socioeconômicos menos favorecidos, pois podem proporcionarlhes melhor consumo de alimentos ricos em nutrientes e energia, ao disponibilizar uma alimentação que a criança não disporia em $\operatorname{casa}^{(7)}$. Todavia, os estudos realizados no Brasil sobre a saúde infantil, tanto em creches quanto aqueles de base populacional, em especial na Região Amazônica, geralmente restringem-se a informações sobre a avaliação antropométrica, sem informações sobre o consumo alimentar, fundamental para melhor compreender o desenvolvimento de carências nutricionais. Sendo assim, o trabalho teve como objetivo avaliar o estado nutricional e o consumo alimentar de crianças assistidas em creches públicas e privadas no município de Manaus, no Amazonas, acreditando-se que os resultados poderão ser úteis para proposição de estratégias que visem melhor adequação da dieta infantil e, consequentemente, melhor estado nutricional.

\section{Método}

Trata-se de um estudo transversal desenvolvido a partir dos dados coletados no estudo multicêntrico intitulado Estimativa da prevalência de inadequação de nutrientes em crianças de diferentes regiōes do Brasil - Estudo Nutri Infância Brasil, realizado em dez cidades brasileiras, entre estas Manaus, cujos dados são aqui analisados.

No estudo multicêntrico, foi prevista amostra por conveniência de 250 pré-escolares de creches públicas e 100 de creches privadas, com crianças entre 24 e 72 meses. Para calcular o número de crianças a serem entrevistadas em cada cidade, estimou-se prevalência de ingestão inadequada de nutrientes de $65 \%$, com margem de erro de $5 \%$ e nível de confiança de $95 \%$. A quantidade de avaliações em cada estrato de creches (pública ou privada) foi baseada no número de matrículas em creches, registrado no Ministério da Educação, Censo Escolar (2005), para os estados brasileiros que participaram do estudo ${ }^{(8)}$. 
Em Manaus, avaliaram-se 217 crianças de duas creches públicas filantrópicas e 91 crianças de duas creches privadas, no período de outubro a dezembro de 2007 , correspondendo a $88 \%$ da amostra prevista no estudo multicêntrico.

Determinou-se o peso em balança eletrônica, portátil, com variação de $50 \mathrm{~g}$, com a criança descalça e com o mínimo de vestimentas. A estatura foi medida utilizando-se antropômetro portátil com escala de $0,1 \mathrm{~cm}$, com a criança descalça, em posição ortostática. O estado nutricional das crianças menores de cinco anos de idade foi classificado com base nos índices peso-para-idade (P/I), peso-paraestatura (P/E), estatura-para-idade (E/I) e índice de massa corporal (peso/estatura ${ }^{2}$ ) para a idade (IMC/I), expressos em valores de escores Z, utilizando como referência os valores da World Health Organization (WHO) $)^{(9)}$. Para as crianças de cinco e seis anos foram utilizados os mesmos índices, com exceção do $\mathrm{P} / \mathrm{E}$ pelo fato da curva de valores para este índice não ter sido disponibilizada na referência da World Health Organization para crianças de cinco a 19 anos de idade ${ }^{(10)}$. Os pontos de corte para classificar déficits e excessos ponderais e estaturais foram baseados nas recomendações do SISVAN $^{(11)}$, as quais são adaptadas da $\mathrm{WHO}^{(9,10)}$.

O consumo alimentar foi obtido por meio do método da pesagem direta de alimentos consumidos pelas crianças em um dia nas creches. Os alimentos servidos no prato da criança foram pesados em balança digital, com capacidade para $3 \mathrm{~kg}$. Foram feitas três pesagens de cada porção de alimento para se obter um valor médio da quantidade de alimentos oferecidos pela creche em cada refeição. Para se determinar o resto alimentar individual, os alimentos deixados no prato por cada criança após a refeição foram pesados. Os restos foram coletados em sacos plásticos identificados para cada criança, sendo pesados sem separação dos alimentos. Para separá-los, utilizou-se o critério de proporcionalidade, ou seja, o percentual de cada alimento oferecido no prato total também foi considerado como percentual de resto e o seu equivalente em gramas. A quantidade de alimentos ingeridos foi considerada a quantidade ofertada menos o resto. A fim de completar o consumo alimentar da criança, os alimentos consumidos fora da creche e respectivas quantidades foram informados pelos pais ou responsáveis pelo método de registro alimentar.

Efetuou-se uma segunda pesagem em 25\% da amostra, selecionada aleatoriamente, tanto nas creches públicas quanto privadas, para avaliar a variância do consumo alimentar das crianças. Esta segunda pesagem foi feita com intervalo de pelo menos um dia da primeira pesagem, repetindo o mesmo procedimento. Ajustou-se a distribuição da ingestão de nutrientes com a remoção do efeito da variância intraindividual, de acordo com o método proposto pelo Iowa State University (ISU) ${ }^{(12)}$, indicado pelo Instituto de Medicina, baseando-se na premissa de que é possível coletar dados de uma subamostra e extrapolar os resultados da estimativa de variância de consumo intraindividual para toda a população avaliada ${ }^{(13)}$. As análises de remoção do efeito da variância intraindividual utilizaram o software PC-SIDE.

A conversão das medidas caseiras em gramas ou mililitros foi feita com base nas padronizações de Pinheiro et al ${ }^{(14)} \mathrm{e}$ Fisberg e Villar ${ }^{(15)}$. Os inquéritos alimentares foram digitados e analisados no programa Nutrition Data System for Research ${ }^{(16)}$, cuja principal base de dados é a tabela norte-americana desenvolvida pelo Departamento de Agricultura dos Estados Unidos (USDA). Os alimentos regionais não existentes no software foram substituídos por alimentos com valores nutricionais semelhantes.

O consumo de nutrientes foi ajustado pelo teor de energia da dieta, pelo método de resíduos proposto por Willet ${ }^{(17)}$, utilizando-se o software STATA versão 10.

Para classificar a adequação da ingestão de energia, utilizou-se a necessidade estimada de energia (Estimated Energy Requirements - EER) ${ }^{(18)}$ para cada criança, de acordo com sexo e idade, considerando-se ingestão excessiva quando ultrapassados $120 \%$ de EER e deficiente quando inferior a $80 \%$. Avaliou-se a proporção de crianças com ingestão de nutrientes abaixo dos requerimentos médios estimados (Estimated Avarage Requirement - EAR) estabelecidos para cada nutriente ${ }^{(19)}$. Quando o nutriente não tinha EAR estabelecido, foram analisados os valores abaixo da ingestão adequada (Adequate Intake - AI). Foram também verificadas as proporções de crianças cujos valores de nutrientes da dieta excediam o limite máximo tolerável de ingestão (Upper Limit - UL) para os nutrientes que possuíam este limite definido ${ }^{(19)}$.

A classificação socioeconômica foi feita a partir do Critério de Classificação Econômica Brasil, utilizando-se o Questionário de Avaliação do Nível Social e Econômico da Associação Brasileira de Empresas de Pesquisas (ABEP) ${ }^{(20)}$, o qual foi autorrespondido pelos responsáveis das crianças e leva em consideração a presença e quantidade de eletrodomésticos e empregados no domicílio e a escolaridade 
do chefe da família. De acordo com o critério da ABEP, as classes econômicas são divididas em A1, A2, B1, B2, $\mathrm{C}, \mathrm{D}$ e $\mathrm{E}$ (da classe mais alta para a mais baixa). Para este estudo, agruparam-se as classes A1 e A2, B1 e B2, D e E, resultando em classes: A, B, C e D.

$\mathrm{Na}$ análise estatística, avaliou-se, inicialmente, a natureza de distribuição das variáveis pelo teste Komogorov-Sminorv. As variáveis contínuas foram descritas em média e erro padrão e as categóricas, em frequência. As comparações das médias entre creches públicas e privadas foram feitas pelo teste $t$ de Student ou teste de Mann-Whitney, dependendo da natureza das suas distribuições, e a comparação das frequências foi feita pelo qui-quadrado. A análise estatística utilizou o software SPSS para Windows, versão 13.0. Foi considerado o valor de $p<0,05$ para significância estatística.

O projeto do estudo multicêntrico foi aprovado pelo Comitê de Ética da Unifesp.

\section{Resultados}

A proporção de crianças com quatro anos ou mais foi maior nas creches públicas $(60,8$ versus $24,1 \%)$, enquanto a proporção de crianças menores de quatro anos foi maior nas creches privadas $(75,8$ versus $39,1 \%)$. Como era esperado, nas instituições privadas prevaleceram crianças pertencentes às classes $\mathrm{A}(62,2 \%)$ e B $(32,2 \%)$, enquanto nas públicas os pré-escolares pertenciam principalmente às classes $\mathrm{C}(64,9 \%)$ e D $(26,7 \%)$ (Tabela 1$)$.
Com base nos índices P/I e P/E, verificou-se maior proporção de crianças das creches privadas com excessos e maior proporção das crianças das creches públicas com déficits ponderais. Quando avaliadas pelo IMC/I, houve maior proporção de excesso de peso nas creches privadas do que nas públicas (Tabela 2).

Quanto à avaliação da dieta consumida (Tabela 3), verificou-se que as crianças das instituições públicas consumiram mais $(p<0,05)$ gorduras poli-insaturadas, ácido graxo ômega- 6 , gordura trans, vitamina $\mathrm{C}$ e sódio do que as crianças das creches privadas.

Com relação à inadequação de consumo energético (Tabela 4), observou-se que, tanto nas creches públicas quanto nas privadas, a proporção de crianças com consumo acima de EER foi cerca de $40 \%$, bem maior do que a proporção com consumo abaixo das recomendações $(5,1$ versus $6,7 \%$, respectivamente). Maior proporção de crianças das creches públicas ingeriram cálcio em quantidades abaixo da AI (27,6 versus 7,9\%, $p<0,001)$, e o consumo de vitamina $\mathrm{D}$ abaixo das recomendações foi igualmente elevado em ambos os grupos (25,8 versus $20,2 \%)$. Nos dois tipos de creches, observou-se proporção elevada de crianças com consumo de vitaminas A e $\mathrm{C}$, zinco e sódio acima do limite superior. Para vitamina A e zinco, estas proporções foram significativamente maiores nas crianças das creches privadas. $\mathrm{O}$ consumo de sódio elevado foi observado em mais de $70 \%$ das crianças investigadas, independentemente do tipo de creche que frequentavam. Todas as crianças apresentaram adequação da ingestão de ferro e estavam com consumo de proteínas acima das recomendações (Tabela 4).

Tabela 1 - Distribuição das crianças das creches públicas e privadas segundo idade, sexo, e classe socioeconômica

\begin{tabular}{|c|c|c|c|c|c|}
\hline & \multicolumn{2}{|c|}{ Públicas (n=217) } & \multicolumn{2}{|c|}{ Privadas $(n=91)$} & \multirow{2}{*}{ Valor $p$} \\
\hline & $\mathbf{n}$ & $\%$ & $\mathrm{n}$ & $\%$ & \\
\hline Idade (anos) & & & & & $<0,001$ \\
\hline $2-4$ & 85 & 39,1 & 69 & 75,8 & \\
\hline $4-7$ & 132 & 60,8 & 22 & 24,1 & \\
\hline Sexo & & & & & 0,174 \\
\hline Masculino & 108 & 49,7 & 53 & 58,2 & \\
\hline Feminino & 109 & 50,2 & 38 & 41,7 & \\
\hline Classificação socioeconômica & & & & & $<0,001$ \\
\hline A & 0 & 0 & 56,0 & 62,2 & \\
\hline B & 18,0 & 8,2 & 29,0 & 32,2 & \\
\hline $\mathrm{C}$ & 141,0 & 64,9 & 5,0 & 5,5 & \\
\hline D & 58,0 & 26,7 & 0 & 0 & \\
\hline
\end{tabular}


Tabela 2 - Distribuição das crianças das creches públicas e privadas, segundo estado nutricional

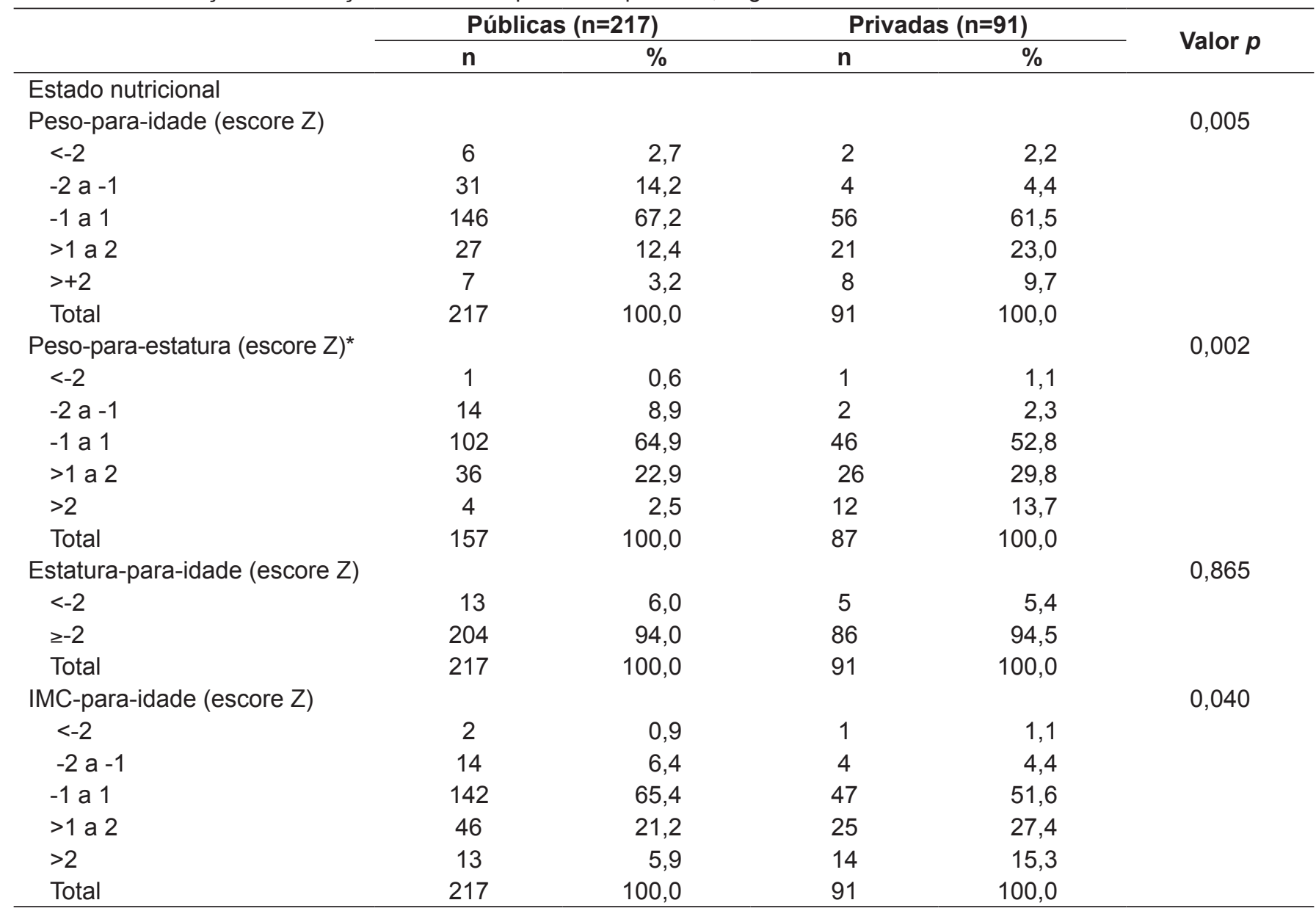

*60 crianças das creches públicas e quatro crianças das creches privadas tinham mais de cinco anos de idade e por isso não foram avaliadas por peso-para-estatura

\section{Discussão}

Este estudo demonstrou existirem diferenças com relação ao estado nutricional entre crianças que frequentam creches públicas e privadas de Manaus, mas as diferenças no consumo alimentar foram menos evidentes. Constatou-se que as crianças que frequentam creches privadas possuem maior tendência ao desenvolvimento de excesso de peso e obesidade do que as crianças das creches públicas, enquanto que as crianças das creches públicas têm maior tendência a apresentar baixo peso tanto para a idade quanto para a estatura.

Em estudo realizado com 216 pré-escolares de Manaus, Marinho et $a^{(21)}$ também constataram maior índice de desnutrição de Grau I (entre 76 e 90\% de adequação de peso para a idade), segundo a classificação de Gomez ${ }^{(22)}$, em crianças de classe socioeconômica baixa, chegando a atingir 47,5\% nos meninos e $48,3 \%$, nas meninas. Desta forma, mesmo considerando as diferenças metodológicas de classificação, pode-se deduzir que, apesar das crianças de condições socioeconômicas mais desfavoráveis estarem em maior risco de déficits nutricionais do que as de estratos mais elevados, aparentemente houve uma redução destes déficits em crianças de Manaus, já que no nosso estudo estas proporções, considerando o índice P/I, não ultrapassaram 18\%.

Quanto ao déficit de E/I, a proporção encontrada (em torno de 6\%) é semelhante à descrita para as crianças brasileiras até cinco anos de idade ${ }^{(2)}$, mas é menor do que a relatada por Alencar et $a l^{(23)}$, que evidenciaram como principal manifestação da desnutrição infantil no Amazonas o déficit de crescimento, que chega a atingir entre 15,6 e 35,2\% das crianças, dependendo do ecossistema da região em que habitam. Tal diferença pode ser atribuída ao fato de estarmos descrevendo o estado nutricional de crianças residentes na área urbana de Manaus, com condições de vida mais favoráveis do que as observadas nestas regiões. 
Tabela 3 - Médias e erros padrão de energia, macro e micronutrientes consumidos na dieta de crianças de creches públicas e privadas

\begin{tabular}{|c|c|c|c|}
\hline \multirow[t]{2}{*}{ Nutrientes } & $\begin{array}{c}\text { Públicas } \\
(n=217)\end{array}$ & $\begin{array}{c}\text { Privadas } \\
(n=89)\end{array}$ & \multirow[t]{2}{*}{ Valor $p$} \\
\hline & Média* (EP) & Média* (EP) & \\
\hline Energia (kcal) & $1.592,0(21,3)$ & $1.564,1(38,5)$ & 0,500 \\
\hline Proteínas $(\mathrm{g})$ & $61,4(1,0)$ & $61,7(1,6)$ & 0,837 \\
\hline \% de proteína em relação ao consumo energético & $15,5(0,2)$ & $15,9(0,2)$ & 0,163 \\
\hline Carboidrato $(\mathrm{g})$ & $233,4(3,4)$ & $228,0(6,1)$ & 0,423 \\
\hline \% de carboidrato em relação ao consumo energético & $58,6(0,3)$ & $58,3(0,5)$ & 0,633 \\
\hline Gordura $(g)$ & $48,6(0,7)$ & $47,3(1,1)$ & 0,315 \\
\hline \% de gordura em relação ao consumo energético & $27,7(0,2)$ & $27,4(0,3)$ & 0,588 \\
\hline Gordura saturada $(\mathrm{g})$ & $18,2(0,3)$ & $18,1(0,5)$ & 0,885 \\
\hline \% de gordura saturada em relação ao consumo energético & $10,3(0,1)$ & $10,5(0,2)$ & 0,461 \\
\hline Gordura monoinsaturada $(\mathrm{g})$ & $15,0(0,2)$ & $14,6(0,4)$ & 0,362 \\
\hline \% de gordura monoinsaturada em relação ao consumo energético & $8,5(0,1)$ & $8,5(0,1)$ & 0,745 \\
\hline Gordura poli-insaturada $(g)$ & $10,4(0,2)$ & $9,7(0,3)$ & 0,018 \\
\hline \% de gordura poli-insaturada em relação ao consumo energético & $5,9(0,1)$ & $5,6(0,1)$ & 0,006 \\
\hline 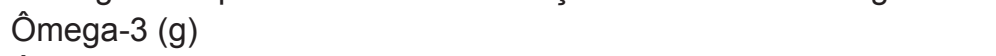 & $1,4(0,0)$ & $1,4(0,0)$ & 0,450 \\
\hline Ômega-6 (g) & $8,7(0,2)$ & $8,0(0,2)$ & 0,015 \\
\hline Gordura trans $(\mathrm{g})$ & $2,6(0,1)$ & $2,1(0,1)$ & 0,008 \\
\hline \% de gordura trans em relação ao consumo energético & $1,4(0,0)$ & $1,2(0,1)$ & 0,008 \\
\hline Vitamina A (mcg) & $602,0(16,4)$ & $609,6(22,1)$ & 0,476 \\
\hline Vitamina D (mcg) & $8,6(0,4)$ & $7,7(0,4)$ & 0,412 \\
\hline Vitamina C (mg) & $1.491,7(153,9)$ & $918,7(134,7)$ & 0,024 \\
\hline Ferro $(\mathrm{mg})$ & $11,8(0,1)$ & $11,9(0,2)$ & 0,842 \\
\hline Cálcio (mg) & $885,5(17,6)$ & $920,0(26,3)$ & 0,286 \\
\hline Zinco (mg) & $8,7(0,1)$ & $9,3(0,2)$ & 0,005 \\
\hline Sódio (mg) & $1.954,6(21,8)$ & $1.828,9(29,0)$ & 0,001 \\
\hline
\end{tabular}

EP: erro padrão; *ajustado pela variância intraindividual e energia

$\mathrm{O}$ excesso de peso, no entanto, foi bastante elevado. Se considerarmos o risco a partir do escore $\mathrm{Z}$ acima de 1 , a frequência chegou a atingir cerca de $25 \%$ das crianças de creches públicas e mais de $40 \%$ das crianças de creches privadas. Estes resultados estão de acordo com o aumento da prevalência de sobrepeso observado no $\mathrm{Brasil}^{(2)}$ e no próprio estudo nacional Nutri Brasil Infância( ${ }^{(3)}$, do qual este trabalho faz parte, reforçando que a obesidade é um problema de Saúde Pública tanto nos países desenvolvidos quanto naqueles em desenvolvimento ${ }^{(24)}$. O consumo energético acima das recomendações em cerca de $40 \%$ das crianças avaliadas pode explicar a elevada frequência de excesso de peso encontrada.

Não obstante as diferenças socioeconômicas e na classificação de estado nutricional entre os tipos de creches, não houve diferença significativa quanto ao consumo de energia e da maioria dos nutrientes entre os dois grupos. Estes achados contrariam a hipótese de que as crianças de creches privadas consumiriam mais energia e nutrientes do que as das creches públicas, por pertencerem a classes socioeconômicas mais altas e, provavelmente, com maior acesso a alimentos. É provável que a frequência às creches esteja contribuindo para um consumo mais adequado de alimentos, independentemente da classe social à qual as crianças pertençam.

Verificou-se consumo abaixo das recomendações apenas de vitamina $\mathrm{D}$ em ambos os tipos de creche e de cálcio nas instituições públicas. O consumo inadequado destes nutrientes pode acarretar comprometimento do crescimento e formação inadequada do tecido ósseo ${ }^{(25)}$. A maior inadequação no consumo de cálcio nas creches públicas pode decorrer da maior proporção de crianças acima de quatro anos neste tipo de creche, as quais já consomem menor quantidade de leite do que as de menor idade.

Outro dado relevante do presente estudo refere-se ao elevado consumo de vitamina $\mathrm{C}$, zinco e sódio (acima da UL) pelas crianças de ambos os grupos. Particularmente o sódio 
Tabela 4 - Frequência de crianças com consumo de energia e nutrientes acima ou abaixo das recomendações em creches públicas e privadas

\begin{tabular}{|c|c|c|c|c|c|}
\hline & Púl & 217) & & 89) & \\
\hline & $\mathrm{n}$ & $\%$ & $\mathrm{n}$ & $\%$ & Valor $p$ \\
\hline Energia & & & & & \\
\hline$<80 \%$ da EER & 11 & 5,1 & 6 & 6,7 & 0,906 \\
\hline$>120 \%$ da EER & 94 & 43,3 & 37 & 41,6 & 0,906 \\
\hline Proteínas & & & & & \\
\hline Abaixo da EAR & - & - & - & - & - \\
\hline Acima da EAR & 217 & 100 & 89 & 100 & - \\
\hline Vitamina A & & & & & \\
\hline Abaixo da EAR & 1 & 0,5 & - & - & - \\
\hline Acima da UL & 48 & 22,1 & 33 & 37,1 & 0,007 \\
\hline Vitamina D & & & & & \\
\hline Abaixo da Al & 56 & 25,8 & 18 & 20,2 & 0,300 \\
\hline Acima da UL & - & - & - & - & \\
\hline Vitamina C & & & & & \\
\hline Abaixo da EAR & 7 & 3,2 & 2 & 2,2 & - \\
\hline Acima da UL & 118 & 54,4 & 41 & 46,1 & 0,186 \\
\hline Ferro & & & & & \\
\hline Abaixo da EAR & - & - & - & - & \\
\hline Acima da UL & - & - & - & - & \\
\hline Cálcio & & & & & \\
\hline Abaixo da Al & 60 & 27,6 & 7 & 7,9 & $<0,001$ \\
\hline Acima da UL & - & - & - & - & \\
\hline Zinco & & & & & \\
\hline Abaixo da EAR & - & - & - & - & \\
\hline Acima da UL & 77 & 35,5 & 66 & 74,2 & $<0,001$ \\
\hline Sódio & & & & & \\
\hline Abaixo da Al & 1 & 0,5 & 1 & 1,1 & - \\
\hline Acima da UL & 154 & 71,0 & 70 & 78,7 & 0,168 \\
\hline
\end{tabular}

EAR: necessidade média estimada; EER: necessidade estimada de energia; Al: ingestão adequada; UL: limite superior tolerável de ingestão

poderá trazer consequências negativas para a saúde infantil e na vida futura, já que o consumo elevado está associado à alta prevalência de hipertensão arterial, considerada um dos maiores problemas de Saúde Pública em adultos ${ }^{(26)}$. A elevada ingestão de sódio, provavelmente, deve-se ao aumento no consumo de alimentos industrializados, ricos neste mineral, hábitos estes que já podem estar sendo incutidos desde a infância.

A vitamina $C$ consumida em excesso pode acarretar efeitos adversos, como diarréia osmótica e distúrbios gastrintestinais, aumento da excreção de oxalato e formação de cálculo renal ${ }^{(25)}$. Todavia, é provável que a ingestão elevada de vitamina $C$ tenha sido observada pelo fato de, no dia da pesagem de alimentos em ambos os tipos de instituições, o cardápio incluir suco de acerola, que é fonte rica deste nutriente, não representando risco à saúde das crianças. A elevada proporção de crianças que consumiram zinco acima da UL pode ter sido consequência do alto consumo de alimentos ricos em proteínas, os quais também são fontes de zinco.

A maioria dos dados no Brasil sobre a avaliação nutricional de crianças é oriunda de estudos realizados em creches públicas devido ao maior espaço cedido para pesquisa por parte das autoridades governamentais. Historicamente, verificam-se também dificuldades de se obter permissão das instituições privadas para realizar estudos em seu domínio. Tal situação contribuiu para que se tenha uma visão parcial da situação nutricional das crianças, visto que as classes socioeconômicas mais elevadas são pouco representadas, dado que as creches públicas costumam ser frequentadas apenas por crianças de classes socioeconômicas menos favorecidas ${ }^{(27)}$. 
Neste estudo, foi possível verificar que o consumo alimentar das crianças das creches públicas e privadas, de um modo geral, não difere muito quanto à composição em energia, macro e micronutrientes, o que corrobora os achados de Bueno, Marchioni e Fisberg ${ }^{(7)}$ acerca dos benefícios para a nutrição de crianças pertencentes a estratos socioeconômicos menos favorecidos que frequentam creches, as quais, em última instância, acabam recebendo alimentos em qualidade mais adequados do que se estivessem em seus domicílios e semelhante ao consumo dos pré-escolares de estratos socioeconômicos mais elevados das instituições privadas. Yuyama et al ${ }^{(28)}$ também constataram que crianças de uma creche pública de Manaus alcançaram as recomendações preconizadas de micronutrientes, diferentemente das crianças ribeirinhas, ratificando a relevância das creches na melhoria do estado nutricional das crianças.

Quanto aos diferentes tipos de gordura, o maior consumo de gordura trans pelas crianças das creches públicas, comparado ao consumo das crianças das creches privadas, pode representar um maior risco para as primeiras de desenvolvimento de doenças crônicas não transmissíveis. Contudo, em ambos os tipos de creches, a média de consumo deste tipo de gordura foi acima da recomendação de, no máximo, $1 \%$ em relação ao valor energético total da dieta ${ }^{(29)}$, ampliando e reiterando este risco para todos os pré-escolares avaliados. Por outro lado, as crianças das creches públicas também consumiram mais gorduras poli-insaturadas e sua fração ômega-6, as quais estão relacionadas a vários benefícios ao organismo, incluindo a prevenção das doenças crônicas ${ }^{(30)}$. $\mathrm{O}$ maior consumo de gorduras para as crianças das creches públicas poderia, talvez, ser explicado pela diferença de idade entre as crianças dos dois tipos de creches, já que nas creches públicas prevaleceram crianças maiores de quatro anos e, nas creches privadas, menores de quatro anos. Crianças de maior idade consomem maior quantidade de porções de alimentos e, consequentemente, de energia e nutrientes. Todavia, como o consumo de nutrientes foi ajustado pela quantidade de energia da dieta, acreditamos que a diferença etária entre os tipos de creche não tenha influenciado estes resultados.

Entre as limitações deste estudo, pode-se citar o método de registro alimentar utilizado para mensurar o consumo alimentar das crianças fora das creches, o qual depende da cooperação dos entrevistados e exige certo grau de escolaridade. Para minimizar os erros de registro, foram dadas explicações prévias para o preenchimento do formulário e foi feita conferência no momento da entrega dos mesmos pelos responsáveis das crianças. A pesagem dos alimentos realizada em apenas um dia na creche também é uma limitação, pois pode não refletir a variação dos cardápios, apesar desta limitação ter sido atenuada pela avaliação de um segundo dia de consumo em uma subamostra de $25 \%$, para ajustar a ingestão pela variância intraindividual no consumo. Por fim, a amostragem das creches foi selecionada por conveniência e não de forma aleatória, comprometendo a validade externa do estudo. Porém, é provável que as crianças que frequentam creches públicas e particulares em Manaus não difiram muito das que foram avaliadas neste estudo.

Concluindo, foi encontrada baixa frequência de crianças com peso menor do que o adequado para a idade e de estatura para a idade, tanto nas instituições públicas quanto nas privadas. Por outro lado, observou-se elevada frequência de pré-escolares com excesso de peso, principalmente nas creches privadas. $\mathrm{O}$ consumo de macro e micronutrientes, de um modo geral, não diferiu entre pré-escolares que frequentam creches públicas e privadas, com exceção de gorduras poli-insaturadas totais, ácidos graxos ômega-6, gordura trans, vitamina $\mathrm{C}$ e sódio, mais consumidos nas creches públicas, e zinco, nas privadas.

A fim de prevenir o ganho de peso excessivo e suas consequências nos pré-escolares assistidos em creches, recomenda-se atenção para a ingestão elevada de energia observada em grande proporção de crianças, principalmente nas creches privadas, e também para um melhor monitoramento quanto ao consumo de sódio, que se encontrou acima das recomendações. Tais cuidados poderão contribuir para a prevenção, desde a infância, de doenças crônicas não transmissíveis, como obesidade e doenças cardiovasculares, que constituem grandes problemas de Saúde Pública na vida adulta.

\section{Agradecimentos}

À Priscila Maximino pela coordenação operacional do Projeto Nutri Infância Brasil. Ao Guilherme de Pádua Rodrigues, por facilitar a organização logística e coordenação financeira do Projeto Nutri Infância Brasil. À Danone Research pela parceria administrativa e pelo apoio para treinamento das equipes. Aos coordenadores e participantes regionais do projeto Nutri Infância Brasil pelo apoio na coleta dos dados locais. 


\section{Referências bibliográficas}

1. Castro TG, Novaes JF, Silva MR, Costa NM, Franceschini SC, Tinôco AL et al. Characteristics of dietary intake, socioeconomic environment and nutritional status of preschoolers at public kindergartens. Rev Nutr 2005;18:321-30.

2. Brasil. Instituto Brasileiro de Geografia e Estatística. Pesquisa de Orçamento familiar 2008-2009: antropometria e análise do estado nutricional de crianças, adolescentes e adultos no Brasil. Rio de Janeiro: IBGE; 2010 [cited 2010 Jan 10]. Available from: www.ibge.gov.br

3. Fisberg M, Fisberg RM, Maximiano P, Bueno M, Rodrigues GP. Brazilian National Survey of food intake and nutritional status of children 2-5 years NUTRI INFANCIABRASIL project. Abstract of the $19^{\text {th }}$ International Congress of Nutrition; 2009 October 4-9; Bangkok, Thailand. Ann Nutr Metab. Switzerland: Karger. 2009;55:299.

4. Brasil. Ministério da Saúde. Centro brasileiro de análise e planejamento. Pesquisa nacional de demografia e saúde da criança e da mulher - PNDS 2006 [cited 2008 Nov 4]. Available from: http://bvsms.saude.gov.br/bvs/pnds/ img/relatorio_final_pnds2006.pdf

5. Muniz PT, Castro TG, Araújo TS, Nunes NB, da Silva-Nunes M, Hoffmann $\mathrm{EH}$ et al. Child health and nutrition in the Western Brazilian Amazon: population-based surveys in two counties in Acre state. Cad Saude Publica 2007;23:1283-93.

6. Rocha YR, Yuyama LK, Nascimento OP. Nutritional profile of pre-school children and other school children, residents in Palmeira do Javari, AM. Acta Amaz 1993;23:9-14.

7. Bueno MB, Marchioni DM, Fisberg RM. Changes in the nutritional status of children in public day care facilities in the municipality of São Paulo, Brazil. Rev Panam Salud Publica 2003;14:165-70.

8. INEP - Instituto Nacional de Estudos e Pesquisas Educacionais Anísio Teixeira. Resultados do Censo Escolar 2005. Brasília: INEP, 2006 [cited 2011 Jul 15]. Available from: http://www.educacenso.mec.inep.gov.br/web/guest/basicacenso-escolar-sinopse-sinopse

9. World Health Organization. WHO Child Growth Standards: Length/heightfor-age, weight-for-age, weight-for-length, weight-for-height and body mass index-for age: methods and development. Geneva: WHO; 2006 [cited 2009 Jul 02]. Available from: http://apps.who.int/bookorders/anglais/detart1.jsp?se sslan $=1$ \& codlan $=1$ \& codcol $=15 \&$ codcch $=660$

10. de Onis M, Onyango AW, Borghi E, Siyam A, Nishida C, Siekmann J. Development of a WHO growth reference for school-aged children and adolescents. Bull World Health Organ 2007;85:660-7.

11. Brasil. Ministério da Saúde. Secretaria de atenção à saúde - departamento de atenção básica. Protocolos do Sistema de Vigilância Alimentar e Nutricional - SISVAN na assistência à saúde. Brasília: Ministério da Saúde, 2008.

12. Guenther PM, Kott PS, Carriquiry AL. Development of an approach for estimating usual nutrient intake distributions at the population level. J Nutr 1997;127:1106-12.

13. Dwyer J. Avaliação do consumo alimentar. In: Shils ME, Olson JA, Shike M, Ross AC, editors. Tratado de Nutrição moderna na saúde e na doença. $9^{\text {th }}$ ed. São Paulo: Manole; 2003. p.1001-26.

14. Pinheiro AB, Lacerda EM, Benzecry EH, Gomes MC, Costa VM, editors.
Tabela para avaliação de consumo alimentar em medidas caseiras. $5^{\text {th }}$ ed. São Paulo: Atheneu; 2004.

15. Fisberg RM, Villar BS. Manual de receitas e medidas caseiras para cálculo de inquéritos alimentares. São Paulo: Signus; 2002.

16. University of Minnesota, editor. Food and nutrient database and interview system descriptive. Nutrition Coordinating Center, 2009 [cited 2011 Jul 14]. Available from: http://www.ncc.umn.edu/ndsrsupport/ndsrmanual2009.pdf

17. Willett W, editor. Nutritional epidemiology. $2^{\text {nd }}$ ed. New York: Oxford; 1998.

18. Institute of Medicine of the National Academies, editor. Dietary reference intakes for energy, carbohydrate, fiber, fat, fatty acids, cholesterol, protein, and amino acids. Washington: National Academy Press; 2002 [cited 2008 Jan 10]. Available from: http:// www.nap.edu/books/0309085373/html

19. Institute of Medicine of the National Academies, editor. Dietary reference intakes: recommended intakes for individuals, vitamins and elements. Washington: National Academy Press, 2004 [cited 2008 Jan 10]. Available from: http://www.iom.edu/Home/Global/News\%20Announcements/ /media/ Files/Activity\%20Files/Nutrition/DRIs/DRI_Summary_Listing.pdf

20. Associação Nacional de Empresas de Pesquisas. Critério de classificação econômica Brasil. São Paulo: ABEP; 2007 [cited 2008 Jul 08]. Available from: http://www.abep.org/novo/Content.aspx?ContentID=301

21. Marinho HA, Kazapi IM, Fernandes MH, Guedes MR. Avaliação do estado nutricional de pré-escolares de classe sócio-econômica média e baixa da cidade de Manaus: estudo comparativo. Acta Amaz 1992;22:363-8.

22. Gomez F. Desnutrición. Bol Med Hosp Inf Mex 1946;3:543-51.

23. Alencar FH, Yuyama LK, Varejão MJ, Marinho HA. Alimentary insecurity determinants and consequences at Amazonas: ecosystems infuences. Acta Amaz 2007;37:413-8.

24. World Health Organization [homepage on the Internet]. Obesity and overweight. Geneva: WHO; 2003 [cited 2009 May 30]. Available from: http://www.who.int/ hpr/NPH/docs/gs_obesity.pdf

25. Mahan KL, Arlin TM. Vitaminas. In: Mahan LK, Escott-Stump S, editors. Krause - Alimentos, nutrição e dietoterapia. $11^{\text {th }}$ ed. São Paulo: Roca; 2005.

26. Salgado CM, Carvalhaes JT. Arterial hypertension in childhood. J Pediatr (Rio J) 2003;79 (Suppl 1):S115-24.

27. Brunken GS, Guimarães LV, Fisberg M. Anemia in children under 3 years of age in public day care centers. J Pediatr (Rio J) 2002;78:50-6.

28. Yuyama LK, Aguiar JP, Nagahama D, Alencar FH, Fávaro DL, Farias LA et al. Micronutrientes em dietas de pré-escolares de diferentes ecossistemas amazônicos. In: Peters BS, Slater B, Cesar CL, Fávaro DI, Nagahama D, Jesus EF et al. editors. Consumo alimentar de micronutrientes. Série de Publicações ILSI Brasil: Alimentos fortificados e suplementos - v. 3. São Paulo: ILSI Brasil - International Life Sciences Institute do Brasil; 2008. p. 75-8.

29. World Health Organization. Diet, nutrition and the prevention of chronic diseases. Geneva: WHO; 2002 [cited 2010 Dec 30]. Available from: http:// whqlibdoc.who.int/trs/who_trs_916.pdf

30. Martin CA, Almeida VV, Ruiz MR, Visentainer JE, Matshushita M, Souza NE et al. Omega-3 and omega- 6 polyunsaturated fatty acids: importance and occurrence in foods. Rev Nutr 2006;19:761-70. 